\title{
Ocurrencia de eventos de sequías en la ciudad de Santiago de Chile desde mediados del siglo $\mathrm{XIX}^{1}$
}

\author{
Álvaro González-Reyes²
}

\begin{abstract}
RESUMEN
Registros instrumentales extensos de precipitación son de amplia utilidad ya que permiten evaluar el comportamiento de eventos extremos como las sequías a múltiples escalas temporales. La ciudad de Santiago de Chile posee un registro histórico desde el año 1867, siendo uno de los más antiguos y completos de América del Sur. En este estudio se analizó la ocurrencia de sequías definidas por umbrales de precipitación (moderadas, severas y extremas), considerando los meses de abril - octubre entre los años 1867 - 2015. Los resultados muestran un incremento en la ocurrencia de toda clase de sequías desde 1930, y un incremento de sequías consecutivas de 2 a 4 años sin precedentes dentro del periodo instrumental. En adición, un incremento de sequías extremas ha sido registrada desde el año 1950 en los meses de mayo a julio, revelando condiciones invernales más secas para la ciudad de Santiago.
\end{abstract}

Palabras clave: Ocurrencia de sequías, Santiago de Chile, Precipitación, Chile Central.

\begin{abstract}
High quality instrumental precipitation records are useful in evaluating extreme events suchs as droughts and their recurrence rate at different time scales. The city of Santiago de Chile has a historical rainfall record that started in 1867, making it one of the most complete and extensive in South America. In this work, April through October precipitation have been analyzed over the 1867 - 2015 period in order to evaluate moderate, severe and extreme of droughts occurrence rate based on precipitation reductions. Main results expose an increase of all types of droughts since 1930 and an increase of consecutive drought episodes unprecedented over the instrumental period. In addition, there has been an increase in extreme droughts occurring from May to June since 1950 indicanting very dry winter conditions for the city of Santiago, Chile.
\end{abstract}

Key words: Drought occurrence, Santiago de Chile, Precipitation, Central Chile.

\footnotetext{
El autor agradece al personal de la Dirección Meteorológica de Chile DMC que ha mantenido vigente el registro instrumental histórico de temperaturas y precitaciones. Además, agradece a la Corporación Nacional de Ciencia y Tecnología CONICYT por su apoyo doctoral y a los revisores anónimos por sus comentarios y sugerencias. Artículo recibido el 26
}

de enero de 2016, aceptado el 6 de junio de 2016 y corregido el 1 de julio de 2016.

2 Departamento de Geología, Universidad de Chile (Chile) / Instituto de Ciencias de la Tierra, Universidad Austral de Chile (Chile).

E-mail: gonzalezreyesalvaro@gmail.com 
La sequía es una característica natural del clima y ocurre en casi todas las regiones climáticas, variando su frecuencia, severidad y duración (Wilhite, 1993). Además, la sequía es considerada una amenaza natural y un desastre al mismo tiempo (Paulo et al., 2012). Se considera una amenaza debido que es un fenómeno impredecible, y un desastre debido a los impactos que provoca sobre los ecosistemas naturales (e.g. incendios forestales, contaminación de las aguas superficiales y subsuperficiales, pérdida de humedad y desnutrición de los suelos, disminución de calidad de las aguas), y el desarrollo de actividades humanas (e.g. abastecimiento de agua potable, hidroelectricidad, agricultura). La definición de sequía per se es compleja y variada, sin embargo se puede entender como un desbalance temporal en la cantidad de agua en base a reducciones en las precipitaciones bajo un valor promedio, cuya frecuencia se desconoce al igual que su duración, resultando una menor disponibilidad en los recursos hídricos (Pereira, 2009).

Los registros instrumentales extensos y continuos de precipitación son una útil herramienta para evaluar fluctuaciones de eventos extremos como las sequías a múltiples escalas temporales (e.g. años, décadas y multidécadas). Esto toma aún una mayor relevancia en latitudes que presentan una marcada estacionalidad de las precipitaciones y un periodo estival prolongado, como es el caso de la ciudad de Santiago - capital de Chile, donde viven un total de 6.061.185 de habitantes (INE, 2003), y que posee una fuerte actividad económica agroindustrial y minera.

Climáticamente, la variación de las precipitaciones en la ciudad de Santiago y la zona central de Chile $\left(30^{\circ}-37^{\circ} \mathrm{S}\right)$ están estrechamente reguladas por la fluctuación anual en la posición del Anticiclón Subtropical del Pacífico y la actividad del fenómeno del Niño ENSO (Aceituno, 1988; Montecinos \& Aceituno, 2003). Además de ello, el régimen de precipitación de Santiago y Chile Central presenta una conexión con anomalías decadales en la temperatura de superficie del mar ocurridas en la región Pacífico $\left(20^{\circ} \mathrm{N}\right.$ hacia el Polo Norte), denominada Oscilación Decadal del Pacífico PDO (Quintana \& Aceituno, 2012). En los últimos años, la región de Chile Central ha vivido una severa sequía registrada entre los años 2010 - 2014. Esta sequía ha sido provocada por una pro- longada reducción en las precipitaciones con disminuciones de casi un 30\%, lo cual ha generado reducciones en los volúmenes de agua de los principales embalses de Chile Central (e.g. Puclaro, La Paloma, El Yeso), la declaración de zonas de escasez hídrica, y la necesidad de una evaluación a escala país de este particular periodo extremo denominado "Megasequía” (CR2 2015). El patrón espacial que ha tenido la megasequía (desde los $30^{\circ}-40^{\circ} \mathrm{S}$ ), no ha sido observado en sequías anteriores ocurridas en por lo menos desde el año 1950 hacia el presente. Desde un punto de vista hidrológico, la disminución en las precipitaciones durante la megasequía ha generado que las descargas de los principales ríos de Chile Central (e.g. río Choapa, Aconcagua, Maipo, Maule) también hayan sido afectados, disminuyendo sus caudales entre un $20 \%$ a un $50 \%$ si se contrasta el intervalo 2013 - 2014 respecto al caudal promedio (DGA-MOP 2016). Cabe mencionar que esta megasequía, que tiene su origen en la variabilidad natural del clima, tiene además una importante contribución provocada por la actividad antropogénica, la cual es responsable en un $25 \%$ de las reducción de las precipitaciones observadas desde el año 2010 (Bosier et al., 2015).

Los estudios de las precipitaciones en Chile Central, incluyendo la precipitación de Santiago, se han focalizado principalmente en la evaluación de sus tendencias (Quintana 2004), fluctuaciones decadales (Quintana \& Aceituno 2012), detección de patrones espacio-temporales (Montecinos \& Aceituno 2003), y relación con forzantes climáticos de escala global (Garreaud et al., 2009). Sin embargo, una evaluación de los cambios temporales en la ocurrencia de eventos extremos de sequías en los meses de mayor aporte pluviométrico aún no ha sido desarrollado. En este sentido, el presente estudio tuvo como objetivo principal evaluar la ocurrencia de sequías en los meses de abril a octubre de la ciudad de Santiago de Chile, basado en el registro histórico de precipitación de la estación meteorológica de Quinta Normal entre los años 1867 - 2015.

\section{Metodología}

El área de estudio corresponde a la ciudad de Santiago de Chile ubicada entre los $33^{\circ} 27^{\prime}$ S y $70^{\circ} 40^{\prime} \mathrm{O}$. El clima que domina a la Re- 
gión Metropolitana, región donde está inserta la ciudad de Santiago, se caracteriza por una marcada concentración de las precipitaciones en los meses de invierno (mayo a agosto) y una marcada y prolongada estación estival seca (Miller, 1976). La variabilidad de las precipitaciones en Chile Central está fuertemente relacionada con la actividad del fenómeno del Niño (ENSO; Aceituno, 1988). La actividad de ENSO, bajo una fase cálida o "El Niño", genera incrementos sobre la media en la pluviosidad de invierno en Chile Central (Rutland \& Fuenzalida, 1991; Montecinos \& Aceituno, 2003). Contrariamente, bajo un condición fría o "La Niña" de ENSO, una pluviosidad menor a la media es registrada en la estación invernal. Aparte de ENSO, algunos estudios señalan que modos de variabilidad decadal a interdecadal como la Oscilación Decadal del Pacífico PDO afectarían el régimen de precipitación y la variabilidad hidroclimática en Chile Central (Masiokas et al., 2010; Quintana \& Aceituno, 2012).

\section{Evaluación de eventos de sequías}

Para evaluar la ocurrencia de eventos de sequías se utilizó la información instrumental de precipitación histórica de la estación de Santiago - Quinta Normal, registro que ha sido compilado y manejado por la Dirección Meteorológica de Chile (DMC). Esta esta- ción excepcionalmente presenta mediciones instrumentales ininterrumpidas desde el año 1867 hasta la fecha. Este extenso registro pluviométrico es además parte de las mediciones históricas de pluviometría de Sudamérica, y en conjunto a otras estaciones meteorológicas de extensa medición (e.g. Fortaleza, Río de Janeiro, Valdivia), ha permitido evaluar episodios históricos de la actividad ENSO ocurridos entre 1877-1878, con consecuencias devastadoras a nivel global debido a una intensificación de condiciones de sequía en Asia y África, generando la muerte de más de 20 millones de personas (Aceituno et al., 2008). La calidad de esta estación meteorológica ha sido corroborada por estudios paleoclimáticos de reconstrucción de la precipitaciones de Santiago de Chile desde el año 1220 en base a anillos de crecimiento arbóreo (Boninsegna, 1988), y para todo Chile Central en el último milenio (Le Quesne et al., 2006).

La ocurrencia de eventos de sequías fue calculada en base a la precipitación acumulada de abril a octubre $(\mathrm{AO})$ y en cada mes, representando las condiciones de otoño, invierno y principios de primavera. El motivo de considerar esta combinación de meses (abril - octubre), radica en que en ellos se concentra la mayor porción de agua caída, independiente del periodo considerado para calcular la distribución mensual de las precipitaciones (Figura $N^{\circ} 1$ ).

Figura $\mathrm{N}^{0} 1$

Distribución mensual de la precipitación de la ciudad de Santiago basado en la media obtenida utilizando diferentes intervalos temporales ( 1867 - 2015, 1900 - 2015, 1950 - 2015, 2000 - 2015 y 2010 - 2015). Las unidades de la precipitación están en milímetros. La flecha horizontal en el eje $\mathrm{x}$ es utilizada para resaltar los meses de abril a octubre $(\mathrm{AO})$ considerados para el análisis.

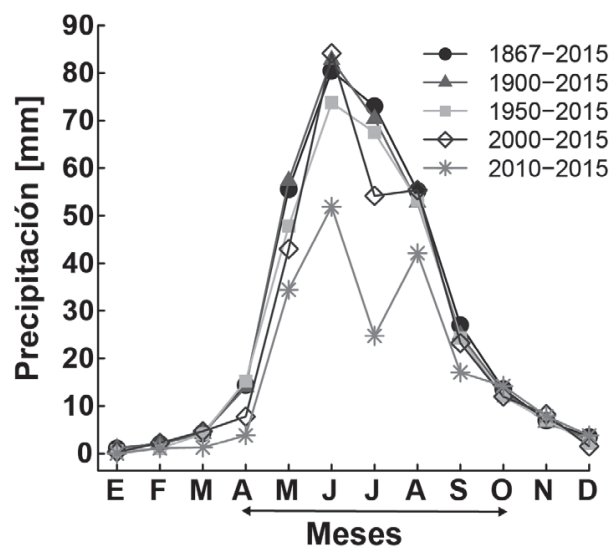

Fuente: Elaboración propia. 
Se establecieron tres tipos de sequías utilizando valores umbrales definidos por valores de percentiles estimados sobre todo el periodo de análisis (1867 - 2015; n = 149 años). Las sequías fueron clasificadas en: moderadas, severas y extremas. Las sequías moderadas fueron aquellas cuyo valor de precipitación fue menor o igual al percentil 25 (e.g. 200 $\mathrm{mm}$ para la precipitación de AO). Las sequías severas fueron aquellas cuyo valor fue menor o igual al percentil 15 (e.g. 173,1 mm para la precipitación de AO), y fueron consideradas sequías extremas a valores anuales menores o iguales al percentil 05 (e.g. 137,1 mm entre AO). A cada serie de eventos obtenida se le calculó la tasa de ocurrencia basado en el método de Kernel. Este método no paramétrico desarrollado por Diggle (1985), permite detectar tendencias no lineales en una serie de eventos estocásticos y permite una suavización más realista de eventos extremos como las sequías. Una función gaussiana y un ancho de banda han sido utilizados para una mejor eficiencia en el proceso de suavización de la tasa de ocurrencia (Mudelsee et al., 2006). Adicionalmente, se le calculó a cada tasa de ocurrencia una banda de confianza a un $P<0,05$ basada en 1000 simulaciones usando bootstrap en orden de representar cambios en la varianza (Cowling et al., 1996). Este método para evaluar eventos extremos ha sido utilizado por diversos autores, por ejemplo, para evaluar inundaciones en ríos de Portugal (Silva et al., 2012), y eventos de paleo-sequías en el Altiplano (Morales et al., 2015) y Chile Centro Sur (Christie et al., 2011; Muñoz et al., 2016). El cálculo de la tasa de ocurrencia de sequías se ha hecho utilizando el paquete estadístico "Paleofire" (Blarquez et al., 2014), escrito para el software de código abierto R - Project (R Core Team 2016), disponible libremente en Internet.

\section{Variaciones en los montos de precipitación y ocurrencia de eventos de sequías en la ciudad de Santiago entre 1867 - 2015}

La precipitación de $\mathrm{AO}$ registra una media histórica de 319,3 mm entre los años 1867 2015 ( $n=149$ años). Años de alta pluviosidad respecto a la media histórica son observados particularmente entre finales del siglo XIX y la primera mitad del siglo XX (Figura $\mathrm{N}^{\circ} 2 \mathrm{~A}$ ). La ocurrencia de eventos de sequías moderadas definidas por el percentil 25 (200 mm) muestra un decrecimiento entre los años 1867 1925 , tomando valores de ocurrencia de entre 3,5 a 6 años, respectivamente. Un incremento en la ocurrencia de sequías moderadas es registrado desde el año 1930 hacia el presente, obteniendo un valor cercano a 3 años entre 2011 - 2015, y una concentración de cuatro eventos consecutivos (Figura $N^{\circ} 2 \mathrm{~B}$ ). No obstante, una concentración particular de diez eventos de sequías moderadas son registrados entre los años 1955 - 1980. La ocurrencia de sequías severas establecidas por el percentil $15(173,1 \mathrm{~mm})$ registra una similar distribución temporal que los eventos definidos por el percentil 25. Valores de ocurrencia cercanos a 5 años son obtenidos entre los años 1869 1872, llegando a valores de 12 años en 1911 y 1924 (Figura No 2C). Una tendencia positiva en la ocurrencia de sequías severas es observada hasta el año 2000, con una tasa de ocurrencia de 4,5 años. Solo dos eventos de sequías severas son registrados entre el periodo 2001 - 2015, uno en el año 2007 y en el año 2011. La ocurrencia de sequías extremas definidas por el percentil $05(137,1 \mathrm{~mm})$ registra una tasa de ocurrencia de entre 20 a 25 años al considerar el periodo 1867 - 1925, seguido de un incremento severo hacía el año 2015, y con valores de ocurrencia de aproximadamente 12 años (Figura $N^{\circ}$ 2D). Una mayor concentración de eventos de sequías de todas las magnitudes es observada particularmente en la segunda mitad del siglo XX y principios del XXI, al contrastarlo con la segunda mitad del siglo XIX y principios del siglo XX. 
Figura $\mathrm{N}^{\circ} 2$

A) Precipitación anual acumulada de abril - octubre $(\mathrm{AO})$ de la ciudad de Santiago entre el periodo 1867 - 2015. La línea continua representa la precipitación media de largo plazo para la ciudad de Santiago, calculada entre 1867- 2015. Las líneas segmentadas corresponden al umbral establecido por los percentiles p05 a p25 utilizados para estimar la tasa de ocurrencia de sequías entre 1867 - 2015. B) ocurrencia de eventos de sequías $\leq$ p25 (200 mm), c) eventos de $\leq$ p15 (173,1 mm), y d) eventos $\leq$ p05 $(137,1 \mathrm{~mm})$. La banda de confianza estadística a un $p<0.05$ calculada en b, c, y d y mostrada en gris, ha sido obtenida en base a 1000 simulaciones utilizando bootstrap. Líneas verticales en la parte inferior de los paneles b, c y d representan los eventos considerados para estimar la ocurrencia de eventos de sequías basados en los percentiles.

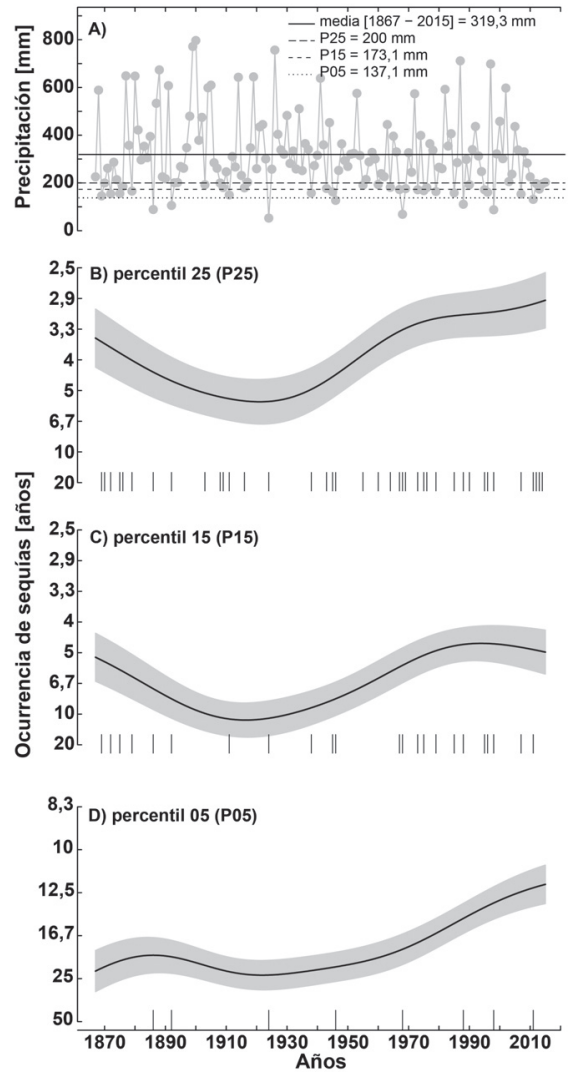

Fuente: Elaboración propia.

\section{Eventos de sequías consecutivas}

La ocurrencia de sequías consecutivas de 2 - 4 años basados en el percentil 25 presentan diferencias en su distribución temporal entre los años 1867 - 2015. Las sequías consecutivas de dos años registran valores de ocurrencia de 12,5 años, y con eventos ocurridos entre 1869 y 1870 . Los valores de ocurrencia decrecen hasta el año 1925, donde se registra una ocurrencia de 33 años continuado con un in- cremento hasta el año 2015, Ilegando a tomar valores de 14 años (Figura $N^{\circ} 3$ ). Las sequías consecutivas de 3 años registran un incremento en los valores de ocurrencia desde 1930, con una concentración de tres eventos 1967 - 1969 y 2011 - 2014, siendo este último intervalo el que presenta cuatro eventos consecutivos. Este intervalo de cuatro años consecutivos de sequías moderadas no registra tener precedentes dentro del registro instrumental que tiene la estación Santiago Quinta Normal. 
Figura $\mathrm{N}^{\circ} 3$

Ocurrencia de sequías consecutivas de 2, 3 y 4 años basado en el percentil 25 (sequías moderadas).

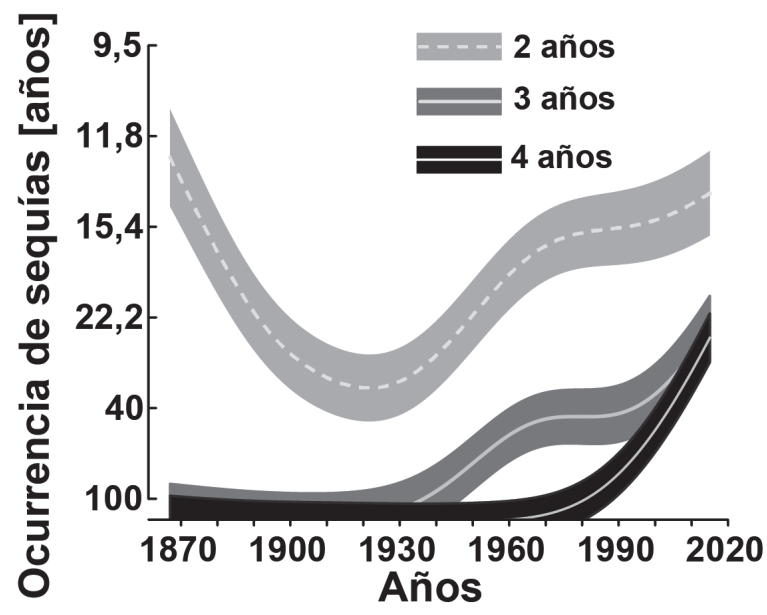

Fuente: Elaboración propia.

\section{Ocurrencia de sequías a escala mensual}

Los meses de abril a octubre muestran diferentes tendencias en la ocurrencia de eventos de sequías moderadas, severas y extremas (Figura $\mathrm{N}^{\circ} 4$ ). El mes de abril registra una similar distribución temporal y tendencia en la ocurrencia de sequías moderadas a lo largo del periodo de análisis, con valores de ocurrencia de entre 3 a 4,5 años (Figura $\mathrm{N}^{\circ}$ 4A). La ocurrencia de sequías moderadas en el mes de mayo muestra una similar distribución temporal entre 1867 - 1930, al igual que la ocurrencia de sequías severas (Figura $N^{\circ}$ 4B). En adición, un incremento en la tasa de ocurrencia de sequías moderadas y severas es observada particularmente desde 1940 al presente, con valores de ocurrencia de entre 2,5 a 4 años al año 2015, respectivamente. Un incremento similar desde 1940 es observado en la ocurrencia de sequías extremas, llegando a valores de ocurrencia de 15 años el año 2015. Los eventos de sequías en el mes de junio muestran similitudes en su tendencia (negativa) entre 1867 - 1920 (Figura $N^{\circ} 4 C$ ). No obstante, un incremento en la ocurrencia de sequías extremas es observada particularmente desde 1920, con valores de ocurrencia que llegan a 9,5 años el año 2015. En el mes de julio se registra un incremento en la ocurren- cia de sequías moderadas y extremas desde el año 1965 ( Figura No 4D). Las sequías moderadas registran valores de ocurrencia de 3,3 a 4 años, mientras que eventos de sequías extremas reportan valores de entre 13 a 25 años. Un intervalo con condiciones de sequías extremas es observado en julio entre los años 1900 - 1920, donde el valor de ocurrencia fue de 15 años. La ocurrencia de eventos de sequías en agosto muestra una condición similar a lo largo del periodo de análisis (Figura $\mathrm{N}^{\circ}$ $4 \mathrm{E})$. No obstante, un leve incremento es registrado en la ocurrencia de sequías extremas entre 1960 - 2015, con valores de ocurrencia de 24 a 17 años, respectivamente. En el mes de septiembre se registra una mayor ocurrencia de sequías severas y extremas concentradas entre los años 1867 - 1930 (Figura № 4F). En adición, un incremento en la ocurrencia de sequías moderadas es observada desde 1930 en adelante, con valores de ocurrencia de 3,5 años entre los años 1968 - 1970. La ocurrencia de sequías moderadas en el mes de octubre registra un decrecimiento entre los años 1867 - 1930, y valores de ocurrencia de 4,5 años (Figura $N^{\circ} 4 \mathrm{G}$ ). No obstante, un incremento en la ocurrencia de sequías moderadas es registrado posterior al año 1930, tomando valores de ocurrencia de 2,6 años el año 2015. 
Ocurrencia de eventos de sequías en los meses: A) abril, B) mayo, C) junio, D) julio, E) agosto, F) septiembre y G) octubre. El ancho de banda utilizado ha sido de 25 años. El doble eje y a la derecha es mostrado en las cajas B - F, y representa la ocurrencia de eventos para el percentil 05. La media histórica correspondiente al periodo 1867 - 2015 es reportada entre paréntesis a continuación del mes respectivo. Las unidades $\mathrm{mm}$ representan milímetros de agua caída. Los valores de los percentiles utilizados para calcular la ocurrencia de sequías moderadas ( $p$ 25), severas (p15) y extremas (p05) son reportado para cada mes en particular.
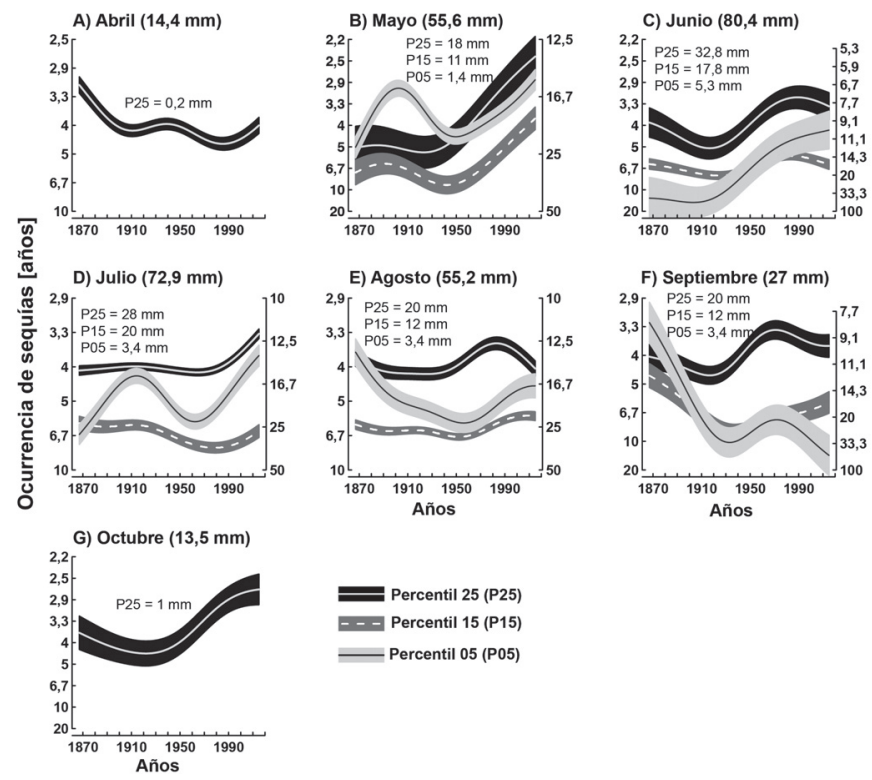

Fuente: Elaboración propia.

\section{La Oscilación Decadal del Pacífico y su influencia en la distribución temporal de eventos de sequías de Santiago de Chile}

La precipitación instrumental de Santiago de Chile se ha analizado desde sus primeras mediciones instrumentales, permitiendo estudiar 149 años de registro pluviométrico ininterrumpido y de excelente calidad debido al arduo trabajo de medición y compilación hecho por la Dirección Meteorológica de Chile. Los resultados obtenidos muestran que la precipitación acumulada de abril a octubre presenta una fuerte variación a lo largo de todo el periodo de análisis, y una marcada distribución temporal de los eventos de sequías. Condiciones particularmente húmedas basados en los años sobre el promedio histó- rico se observan en intervalos temporales específicos (1867 - 1930 y 1970 - 2000; Figura $\mathrm{N}^{\circ}$ 2A). Contrariamente, condiciones secas son registradas desde la década de 1930 1940 hacia el presente, en donde la ocurrencia de las tres clases de sequías (moderadas, severas y extremas), muestran un incremento (Figura $\left.\mathrm{N}^{\circ} 2 \mathrm{~B}-\mathrm{D}\right)$. Este mismo patrón es observado en la ocurrencia de eventos consecutivos de sequías moderadas usando 2 a 4 años, destacándose los eventos entre 2011 - 2014 los cuales no tienen precedentes (Figura $\mathrm{N}^{\circ}$ 3). Los resultados exhiben una predominante reducción de la precipitación acumulada de abril a octubre en la ciudad de Santiago de Chile desde el año 1930 hacia el presente. Esta condición seca es también observable en otras ciudades de Chile. Un ejemplo de ello es el registro pluviométrico histórico de la ciudad de Valdivia ubicada en la zona sur de Chile, donde los cambios en la precipitación anual acumulada muestran una reducción 
significativa desde el año 1853 (GonzálezReyes y Muñoz, 2013). Adicionalmente, un incremento hacia condiciones secas ha sido también registrado en el río Maule desde el año 1850 y desde el año 1920 en el río Biobío, si se consideran las reconstrucciones paleohidrológicas de ambos ríos en base a anillos de crecimiento de árboles (Muñoz et al., 2016).

Autores como Quintana \& Aceituno (2012) describen la existencia de una modulación decadal de las precipitaciones en Chile Central provocada por la Oscilación Decadal del Pacífico PDO. En adición a ello, estudios desarrollados por Masiokas et al. (2010) muestran una coherencia temporal y estrecha relación entre variables hidroclimáticas de Chile Central (e.g. acumulación de nieve y caudales de ríos) con la actividad decadal de la PDO dentro del siglo XX. El índice de la PDO, basado en el estudio de Mantua et al. (1997), exhibe una marcada variabilidad temporal y dos fases bien marcadas a lo largo del siglo XX. Una fase positiva (cálida) es registrada entre los intervalos 1925 - 1944 y 1976 - 1990, mientras que una fase negativa o fría es identificada entre los años 1946 - 1975. Si se contrasta el índice de la PDO con los eventos de sequías identificados desde 1900, y se consideran los valores de ocurrencia registrados especialmente para la sequías extremas (Figura $N^{\circ} 2 \mathrm{D}$ ), existe una concordancia temporal con la fase fría de la PDO observada durante 1900 - 2015 (Figura $N^{0} 5$ ). Esto indicaría que forzantes climáticos de escala decadal como la PDO modularía la distribución temporal de eventos de sequías moderadas, severas y extremas de la precipitación de Santiago de Chile.

Figura $\mathrm{N}^{\circ} 5$

Índice instrumental de la Oscilación Decadal del Pacífico PDO de enero a diciembre entre 1900 2015 representado en anomalías estandarizadas. En la parte baja del panel los eventos de sequías moderadas, severas y extremas encontrados para la precipitación de abril - octubre son mostrados de forma comparativa. La línea horizontal ubicada en 0 representa la división entre la fase fría (valores negativos) y la fase cálida de la PDO.

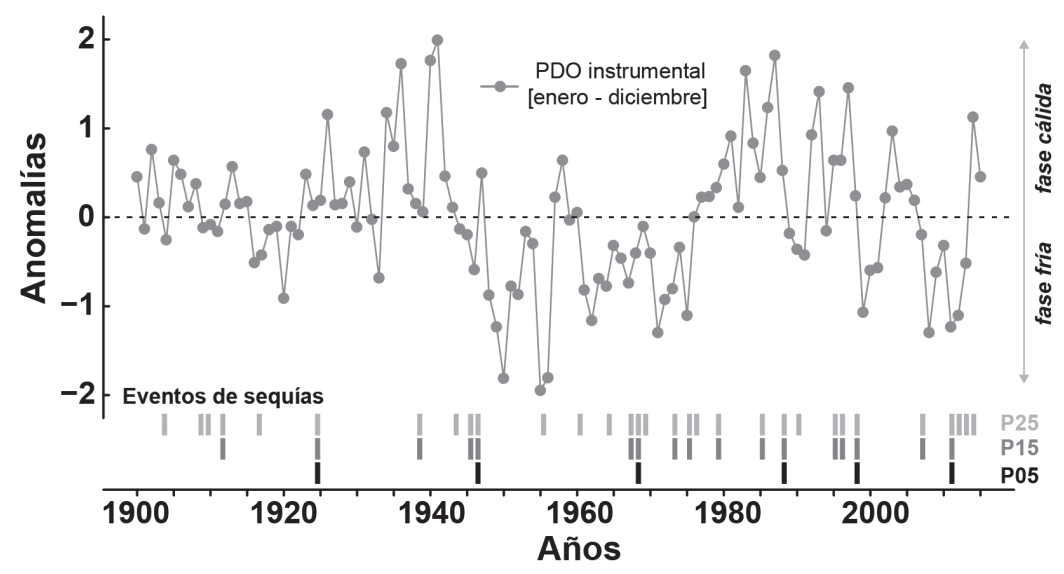

Fuente: Elaboración propia.

Diversos estudios han documentado la estrecha relación entre la actividad de ENSO de los meses de invierno y la precipitación de Chile Central, incluyendo la estación Santiago - Quinta Normal (Rutland \& Fuenzalida, 1991; Montecinos \& Aceituno, 2003).
La actividad de ENSO medida a través del Índice Oceánico del Niño (ONI) registra una alternante condición de eventos La Niña y El Niño entre 1950 - 2015 (Trenberth, 2016). Adicionalmente, estudios paleoclimáticos señalan que la actividad de ENSO ha registrado 
una condición anormal en la segunda mitad del siglo XX en comparación a los últimos siete siglos (Li et al., 2013). En el caso de la distribución temporal de los eventos de sequías, la fuerte variabilidad registrada en la actividad ENSO podría estar contribuyendo a una mayor ocurrencia de eventos de sequías moderadas, las cuales se encuentran mayormente distribuidas en la segunda mitad del siglo XX. Sin embargo, una combinación de condiciones La Niña de ENSO sumado a condiciones frías decadales de la PDO proporcionarían una mayor probabilidad de ocurrencia de eventos de sequías en la precipitación de Santiago de Chile. Un ejemplo de ello es el intervalo de eventos de sequías observado entre los años 1965 - 1976 (Figura $\mathrm{N}^{\circ} 2 \mathrm{~B}-2 \mathrm{D}$ ), en este periodo existe una predominancia de eventos la Niña (Trenberth, 2016), dentro de una fase fría de la PDO bien descrita por Mantua et al. (1997).

En una escala mensual, la precipitación de Santiago registra cambios severos en los meses de mayo a julio, con un incremento en las condiciones de sequías extremas registrada desde el año 1950. Este resultado documenta una importante reducción en las precipitaciones invernales en los meses de mayor contribución pluviométrica. En adición, este resultado es también observable tras comparar la distribución mensual de los montos de precipitación utilizando diferentes intervalos temporales expuesto en la Figura $N^{0} 1$, donde se puede observar con claridad la fuerte disminución de las precipitaciones en el mes de julio, dentro del intervalo 2010 - 2015. Interesantemente, la medición para julio del año 2012 fue de 2 mm de agua caída en Santiago. Este valor no es el menor registrado para este mes en particular, ya que en julio del año 1950 se registró $0,1 \mathrm{~mm}$ de agua caída. En ambos años se registraron condiciones secas o La Niña de ENSO. Dentro del intervalo de años 2010 - 2014, las condiciones de ENSO fueron neutras. Sin embargo, el índice Niño 3,4 de ENSO para el año 2015 ha evolucionado hacia condiciones El Niño las que se relacionan con un incremento de las precipitaciones en Santiago. No obstante, el año 2015 se encuentra bajo el promedio histórico (Figura $\mathrm{N}^{\circ} 2 \mathrm{~A}$ ).

Las causas físicas del incremento en la ocurrencia de sequías extremas registrada entre mayo a julio desde la segunda mitad del siglo XX no es clara. En adición a ello, estudios como el de Bosier et al. (2015) han mostrado que la actividad antrópica también contribuye a la generación de sequías. Si bien la precipitación invernal en Chile Central tiene una fuerte relación con la variabilidad de ENSO (Aceituno, 1988; Montecinos \& Aceituno 2003), existen hipótesis alternativas que señalan que forzantes climáticos provenientes de altas latitudes (e.g. La Oscilación Antártica AAO o Modo Anular del Sur SAM) podrían explicar en parte la reducción de las precipitaciones e incremento en las condiciones de sequías en ciudades como La Serena y Ovalle durante el siglo XX (Vuille y Milana, 2007). Este forzante climático ha mostrado una marcada tendencia positiva, la cual es observada desde 1950 en adelante y sin precedentes dentro de los últimos seis siglos (Villalba et al., 2012). Los cambios en los campos de presión al nivel del mar entre las latitudes medias (e.g. Santiago) y altas $\left(60^{\circ} \mathrm{S}\right)$, que están relacionados con la actividad del AAO, juegan un papel importante en la variabilidad interanual de las precipitaciones de abril a septiembre, y en la migración de los sistemas frontales desde las altas latitudes hacia latitudes medias (Quintana \& Aceituno, 2012). Si se contrasta la relación entre la precipitación mensual de mayo, por ejemplo, y el índice Niño 3,4 de ENSO de mayo se obtiene un valor de correlación $r$ de Pearson menor al obtenido tras comparar la precipitación de mayo con una combinación de meses de marzo - abril del índice AAO de Marshall, ambos dentro del periodo 1957 - 2015. El valor de correlación de Pearson es de $r=0,27$ entre la precipitación de mayo y ENSO, y $r=-0,37$ entre la precipitación de mayo y el AAO sin su tendencia lineal, siendo ambos estadísticamente significantes a un $P<0.05$ y $P<0.01$, respectivamente. Esta observación sugiere que la actividad del AAO estaría afectando el régimen de precipitaciones de finales de otoño e invierno en la Ciudad de Santiago. Este resultado respaldaría los resultados reportados por Quintana \& Aceituno (2012), respecto a la modulación de AAO sobre la precipitación de abril a septiembre. Cabe mencionar que la precipitación de Santiago y el AAO muestran una estrecha relación a una escala temporal interanual, al igual que con la actividad de ENSO. Debido a que ambos forzantes influyen a la precipitación en una escala temporal 
similar, investigaciones futuras respecto a determinar las causas físicas del incremento en la ocurrencia de sequías en la ciudad de Santiago y en Chile Central deberían considerar en sus análisis, a parte de la actividad de ENSO y la PDO, el forzamiento provocado por la actividad de la Oscilación Antártica a múltiples escalas temporales (e.g. décadas y multidécadas), y un tratamiento estadístico a fin de eliminar la señal ENSO sobre las precipitaciones y comprender en mayor medida el forzamiento provocado por el AAO.

Los resultados expuestos en este trabajo describen la fuerte amenaza que presenta la ciudad de Santiago de Chile respecto al incremento en la ocurrencia de sequías basado en 149 años de observaciones en los meses de mayor precipitación. Estos resultados pueden ser una herramienta útil si se considera que: i) la ciudad de Santiago tiene una alta densidad poblacional, y ii) una fuerte actividad agroindustrial y minera, ambas actividades económicas demandantes de una gran cantidad de agua. Por otro lado y desde un punto de vista hidrológico, el incremento hacia condiciones más secas en los meses de mayor aporte de agua se traduce en una respuesta negativa de recursos hídricos estratégicos como es el caso de la acumulación nival y glaciares de montaña. Masiokas et al. ( 2016) documenta la estrecha relación existente entre la acumulación nival de estaciones ubicadas entre los $30^{\circ}-37^{\circ} \mathrm{S}$, los caudales anuales de ríos de Chile y Argentina, el balance de masa del glaciar Echaurren Norte y la precipitación de Santiago, esta última responsable en parte de la notoria reducción de la masa glaciar del Echaurren Norte registrada desde el año 2000. Un entendimiento profundo de los procesos físicos que modulan la precipitación de Santiago, y un mejoramiento en el uso y gestión de los recursos hídricos en la Región Metropolitana serían claves para generar una adaptación realista y eficaz ante condiciones climáticas futuras aún más secas que las actuales en esta ciudad densamente poblada

\section{Conclusiones}

La precipitación acumulada de abril a octubre perteneciente al registro histórico de la ciudad de Santiago de Chile ha sido analizada entre los años 1867 - 2015. Un incremento en la tasa de ocurrencia de sequías moderadas, severas y extremas ha sido registrada desde el año 1930. No obstante, la tasa de ocurrencia de sequías moderadas y extremas muestra valores sin precedentes dentro del registro instrumental, los cuales se encuentran concentrados particularmente desde el año 1950 hacia el presente. Un intervalo de cuatro años consecutivos de sequía moderada es registrado entre los años 2011 - 2014, revelando una condición sin precedentes dentro de 149 años de observación. Condiciones secas son mayormente registradas desde la segunda mitad del siglo XX en la ciudad de Santiago.

Un incremento en la tasa de ocurrencia de sequías extremas en los meses de mayo, junio y julio ha sido registrado desde el año 1950, indicando una condición de sequía invernal desde la segunda parte del siglo $X X$ y principios del siglo XXI para la ciudad de Santiago de Chile. Las condiciones de sequía se han agudizado en el siglo XXI.

El incremento de años e intervalos secos registrado en la precipitación de los meses de invierno en la ciudad de Santiago sugiere focalizar estudios futuros que consideren en sus análisis una evaluación de las precipitaciones a una mayor resolución temporal. En este sentido, una evaluación a escala diaria podría ser de amplia utilidad, considerando la importancia de la precipitación en el ciclo hidrológico (e.g. acumulación nival, recarga de acuíferos, escorrentía superficial), y la fuerte demanda por agua que tiene Santiago para consumo humano y el desarrollo de actividades económicas como la agricultura y la minería.

\section{Referencias bibliográficas}

ACEITUNO, P. On the functioning of the Southern Oscillation in the South American sector. Part I: Surface climate. Monthly Weather Review, 1988, Vol. 116, p. 505-524.

ACEITUNO, P.; PRIETO, M.D.R.; SOLARI, M.E.; MARTÍNEZ, A; POVEDA, G. \& FALVEY, M. The 1877-1878 El Niño episode: associated impacts in South America. Climatic Change, 2008, Vol. 92, p. 389-416. 
BONINSEGNA, J.A. Santiago de Chile winter rainfall since 1220 as being reconstructed by tree rings. Quaternary Of South America And Antarctic Peninsula, 1988, Vol. 6, p. 67-87.

BLARQUEZ, O.; VANNIÈRE, B.; MARLON, J. R.; DANIAU, A.L.; POWER, M.J.; BREWER, S. \& BARTLEIN, P.J. paleofire: an R package to analyse sedimentary charcoal records from the Global Charcoal Database to reconstruct past biomass burning, Computers \& Geosciences, 2014, Vol. 72, p. 255-261.

BOSIER, J.P.; RONDANELLI, R.; GARREAUD, R. \& MUÑOZ, F. Natural and anthropogenic contributions to the Southeast Pacific precipitation decline and recent mega-drought in central Chile. Geophysical Research Letters, 2015, Vol. 43, № 1, p. 413421.

CENTRO DE CIENCIA DEL CLIMA Y LA RESILIENCIA (CR2). La megasequía 20102015: Una lección para el futuro. Santiago de Chile: Centro de Ciencia del Clima y la Resiliencia (CR2), 2015.

CHRISTIE D.A.; BONINSEGNA, J.A.; CLEAVELAND, M.; LARA, A.; LE QUESNE, C.; MORALES, M.; MUDELSEE, M.; STAHLE, D. \& VILLALBA, R. Aridity changes in the Temperate-Mediterranean transition of the Andes since AD 1346 reconstructed from tree-rings. Climate Dynamics, 2011, Vol. 36, p. 1505-1521.

COWLING, A.; HALL, P. \& PHILLIPS, M.J. Bootstrap confidence regions for the intensity of a Poisson point process. Journal of the American Statistical Association, 1996, Vol. 91, p. 1516-1524.

DIGGLE, P. A kernel method for smoothing point process data. Journal of the Royal Statistical Society: Series C (Applied Statistics), 1985, Vol. 34, p. 138-147.

DIRECCIÓN GENERAL DE AGUAS (DGAMOP). Atlas del agua Chile. Santiago de Chile: DGA-MOP, 2016.

GARREAUD, R.; VUILLE, M.; COMPAGNUCCI, R. \& MARENGO, J. Present- day South American climate. Palaeogeography,
Palaeoclimatology, Palaeoecology, 2009, Vol. 281, p.180-195.

GONZÁLEZ-REYES, A. \& MUÑOZ, A. Cambios en la precipitación de la ciudad de Valdivia (Chile) durante los últimos 150 años. Bosque, 2013, Vol. 34, p. 191-200.

INSTITUTO NACIONAL DE ESTADÍSTICAS (INE). Censo Nacional 2002. Santiago de Chile: Instituto Nacional de Estadísticas, 2003.

LE QUESNE, C.; STAHLE, D.; CLEAVELAND. M.; THERRELL, M.D.; ARAVENA, J.C. \& BARICHIVICH, J. Ancient Austrocedrus treering chronologies used to reconstruct central Chile precipitation variability from a.d. 1200 to 2000. Journal of Climate, 2006, Vol. 19 , p. 5731-5744.

LI, J.; XIE, S.P.; COOK, E.; MORALES, M.S.; CHRISTIE, D.; CHEN, F.; D'ARRIGO, R.; JOHNSON, N.C.; FOWLER, A.M.; GOU, X. \& FANG, K. El Niño modulations during the past seven centuries. Nature Climate Change, 2013, Vol.3, p. 822-826.

MANTUA, N.J.; HARE, S.; ZHANG, Y.; WALLACE, J. \& FRANCIS, R.C. A Pacific interdecadal climate oscillation with impacts on salmon production. Bulletin of the American Meteorological Society, 1997, Vol. 78, p. 1069-1079.

MASIOKAS, M.H.; VILLALBA, R.; LUCKMAN, B.H. \& MAUGET, S. Intra - to multidecadal variations of snowpack and streamflow records in the Andes of Chile and Argentina between $30^{\circ}$ and $37^{\circ} \mathrm{S}$. Journal of Hydrometeorology, 2010, Vol. 11, p. 822-831.

MASIOKAS, M. H.; CHRISTIE, D.A; LE QUESNE, C.; PITTE, P.; RUIZ, L.; VILLALBA, R.; LUCKMAN, B.H.; BERTHIER, E.; NUSSBAUMER S.U.; GONZÁLEZ-REYES, A.; MCPHEE, J. \& BARCAZA, G. Reconstructing the annual mass balance of the Echaurren Norte glacier (Central Andes, 33.5 S) using local and regional hydroclimatic data. The Chryosphere, 2016, Vol. 10, p. 927-940.

MILLER, A. The climate of Chile. Climates of Central and South America. Amsterdam: Editorial Elsevier, 1976. 
MONTECINOS, A. \& ACEITUNO, P. Seasonality of the ENSO- related rainfall variability in central Chile and associated circulation anomalies. Journal of Climate, 2003, Vol. 16, p. 281-296.

MORALES, M.; CARILLA, J.; GRAU, H.R. \& VILLALBA, R. Multi-century lake area changes in the Southern Altiplano: a tree-ring-based reconstruction. Climate of the past, 2015, Vol. 11, p. 1139-1152.

MUDELSEE, M.; DEUTSCH, M.; BÖRNGEN, M. \& TETZLAFF, G. Trends in flood risk of the River Werra (Germany) over the past 500 years. Hydrological Sciences Journal, 2006, Vol. 51, 818-833.

MUÑOZ, A.A.; GONZÁLEZ-REYES, A.; LARA, A.; SAUCHYN, D.; CHRISTIE, D.A.; PUCHI, P.; URRUTIA- JALABERT, R.; TOLEDO-GUERRERO, I.; AGUILERA-BETTI, I.; MUNDO, I.; SHEPPARD, P.R.; STAHLE, D.; VILLALBA, R.; SZEJNER, P.; LEQUESNE, C. \& VANSTONE, J. Streamflow variability in the Chilean Temperate-Mediterranean climate transition $\left(35^{\circ} \mathrm{S}-42^{\circ} \mathrm{S}\right)$ during the last 400 years inferred from tree-ring records, Climate Dynamics, 2016, DOI: 10.1007/s00382-0163068-9.

PAULO, A.A.; ROSA, R.D. \& PEREIRA, L.S. Climate trends and behaviour of drought indices based on precipitation and evapotranspiration in Portugal. Natural Hazards and Earth System Sciences, 2012, Vol. 12, p. 1481-1491.

PEREIRA, L.S.; CORDERY, I. \& IACOVIDES, I. Coping with Water Scarcity. Addressing the Challenges. Dordrecht: Springer, 2009.

QUINTANA, J. Estudio de los factores que explican la variabilidad de la precipitación en Chile en escalas de tiempo interdecadal. Santiago de Chile: Tesis para optar al grado de Magíster en Ciencias mención Geofísica, Universidad de Chile, 2004.

QUINTANA, J.M. \& ACEITUNO, P. Changes in the rainfall regime along the extratropi- cal west coast of South America (Chile): 30$43^{\circ}$ S. Atmosfera, 2012, Vol. 25, p. 1-22.

R CORE TEAM A language and environment for statistical computing. Viena: R Foundation for Statistical Computing, 2016.

RUTLLAND, J. \& FUENZALIDA, H. Synoptic aspects of the central Chile rainfall variability associated with the Southern Oscillation. International Journal of Climatology, 1991, Vol. 11, p. 63-76.

SILVA, A.T.; PORTELA, M.M.; \& NAGHETTINI, M. Nonstationarities in the occurrence rates of flood events in Portuguese watersheds. Hidrology and Earth System Sciences, 2012, Vol. 16, p. 241-254.

TRENBERTH, K. The Climate Data Guide: Nino SST Indices (Niño 1+2, 3, 3.4, 4; ONI and TNI). 2016. Disponible en Internet: www.climatedataguide.ucar.edu/climate-data/ nino-sst-indices-nino-12-3-34-4-oni-and-tni.

VILLALBA, R.; LARA, A.; MASIOKAS, M.H.; URRUTIA, R.; LUCKMAN, B.H.; MARSHALL, G.J.M MUNDO, I.; CHRISTIE, D.A.; COOK, E.; NEUKOM, R.; ALLEN, K.; FENWICK, P.; BONINSEGNA, J.A.; SRUR, A.M.; MORALES, M.S.; ARANEO, D.; PALMER, J.G.; CUQ, E.; ARAVENA, J.C.; HOLZ, A. \& LE QUESNE, C. Unusual Southern Hemisphere tree growth patterns induced by changes in the Southern Annular Mode. Nature Geoscience, 2012, Vol. 5, p. 793-798.

VUILLE, M. \& MILANA, J.P. High-latitude forcing of regional aridification along the subtropical west coast of South America. Geophysical Research Letters, 2007, Vol. 34, $N^{\circ} 23$. Disponible en Internet: http://onlinelibrary.wiley.com/doi/10.1029/2007GL031899/ full

WILHITE, D.A. Drought Assessment, Management, and Planning: Theory and Case Studies, Natural Resource Management and Policy Series. Nueva York: Editorial Kluwer Academic, 1993. 